\title{
APLICACIÓN DEL JUEGO SERIO EN PROGRAMAS DE CIENCIAS ECONÓMICAS: TENDENCIAS Y DESAFIOS
}

Morales-Sierra, María Eugenia; Cardona-Valencia, Daniel; Castañeda-Gómez, Eric; Uribe-Ortiz, Ana María; Ríos-Gallego, Paul Alexander

APLICACIÓN DEL JUEGO SERIO EN PROGRAMAS DE CIENCIAS ECONÓMICAS: TENDENCIAS Y DESAFIOS

PANORAMA, vol. 14, núm. 27, 2020

Politécnico Grancolombiano, Colombia

Disponible en: http://www.redalyc.org/articulo.oa?id=343964051008

DOI: https://doi.org/10.15765/pnrm.v14i27.1526

https://journal.poligran.edu.co/index.php/panorama/about/submissions\#copyrightNotice

Esta obra está bajo una Licencia Creative Commons Atribución-NoComercial-SinDerivar 4.0 Internacional. 
PANORAMA, vol. 14, núm. 27, 2020

Politécnico Grancolombiano, Colombia

Recepción: 08 Octubre 2019

Aprobación: 01 Julio 2020

DOI: https://doi.org/10.15765/ pnrm.v14i27.1526

Redalyc: http://www.redalyc.org/ articulo.oa?id $=343964051008$

https://journal.poligran.edu.co/ index.php/panorama/about/ submissions\# copyrightNotice CC BY-NC-ND
Artículos de investigación científica y tecnológica

\section{APLICACIÓN DEL JUEGO SERIO EN PROGRAMAS DE CIENCIAS ECONÓMICAS: TENDENCIAS Y DESAFÍOS}

\author{
APPLICATION SERIOUS GAME IN ECONOMIC \\ SCIENCE PROGRAMS: TRENDS AND CHALLENGES
}

APLICAÇÃO DE JOGOS SÉRIOS EM PROGRAMAS DE CIÊNCIA ECONÔMICA: TENDÊNCIAS E DESAFIOS

María Eugenia Morales-Sierra memorales@poligran.edu.co Institución Universitaria Politécnico Grancolombiano., Colombia

Daniel Cardona-Valencia danielcardona@itm.edu.co Instituto Tecnológico Metropolitano, ITM., Colombia

Eric Castañeda-Gómez ericcastaneda@itm.edu.co Instituto Tecnológico Metropolitano, ITM., Colombia

Ana María Uribe-Ortiz anauribe144623@correo.itm.edu.co Instituto Tecnológico Metropolitano, ITM., Colombia

Paul Alexander Ríos-Gallego paulrios@itm.edu.co Instituto Tecnológico Metropolitano, ITM., Colombia

Resumen: Este documento presenta una caracterización del trabajo colaborativo internacional sobre la aplicación del juego serio en contenidos curriculares de programas en ciencias económicas relacionadas con las finanzas, la contabilidad y la administración. Se tomó como referencia información compilada de la base de datos académica Scopus durante la ventana de observación 2007-2018, teniendo en cuenta indicadores bibliométricos, metodologías destacadas, tendencias de uso o aplicación y desafíos o retos de la implementación. Como resultado, se logra identificar ocho principales categorías de estrategias de enseñanza-aprendizaje en las cuales Aprendizaje adaptativo, Aprendizaje colaborativo y Gamificación se ubican como las tendencias en las que el uso del juego serio se ha consolidado. Storytelling (narrativa de historias), y el Aprendizaje basado en competencias están en proceso de consolidación. Finalmente, Aprendizaje invertido, Massive Open Online Course (MOOC), y Aprendizaje basado en retos son líneas incipientes en las que el uso del juego serio no se ha desarrollado lo suficiente. De igual manera, se logra destacar, a partir del seguimiento de publicaciones científicas, los principales logros, orientaciones y desafíos de la aplicación de estas estrategias en el aula de clase; particularmente, las relacionadas con las finanzas, la administración y la contaduría.

Palabras clave: Educación, juego serio, administración, finanzas y contaduría, aprendizaje activo, Formación por competencias.

Abstract: This document presents a characterization of the international collaborative work on the application of serious game in curricular contents of programs in economics related to finance, accounting and administration. Information compiled from the Scopus academic database during the 2007-2018 observation window was taken as a reference, considering bibliometric indicators, outstanding methodologies, trends in use or application, and challenges or challenges of implementation. As a result, it is possible to identify eight main categories of teaching-learning strategies in which Adaptive 
Learning, Collaborative Learning and Gamification are located as the trends in which the use of serious games has been consolidated. Storytelling (narrative of stories), and Learning based on competencies are in the process of consolidation. Finally, flipped learning, Massive Open Online Course (MOOC), and Challenge-based learning are incipient lines in which the use of serious gaming has not been sufficiently developed. In the same way, it is possible to highlight, from the monitoring of scientific publications, the main achievements, orientations and challenges of the application of these strategies in the classroom; particularly those related to finance, administration and accounting.

Keywords: Education, serious game, administration, finance and accounting, active learning, training by competencies.

Resumo: Este documento apresenta uma caracterização do trabalho colaborativo internacional sobre a aplicação do serious game em conteúdos curriculares de programas de economia relacionados a finanças, contabilidade e administração. As informações compiladas do banco de dados acadêmico Scopus durante a janela de observação 2007-2018 foram tomadas como referência, levando em consideração indicadores bibliométricos, metodologias pendentes, tendências de uso ou aplicação e desafios ou desafios de implementação. Com isso, é possível identificar oito categorias principais de estratégias de ensino-aprendizagem nas quais a Aprendizagem Adaptativa, a Aprendizagem Colaborativa e a Gamificação se situam como as tendências nas quais o uso de serious games se consolidou. Contação de histórias (narrativa de histórias) e Aprendizagem baseada em competências estão em processo de consolidação. Por fim, a aprendizagem invertida, o Massive Open Online Course (MOOC) e a aprendizagem baseada em desafios são linhas incipientes nas quais o uso de jogos sérios não foi suficientemente desenvolvido. Da mesma forma, é possível destacar, a partir do acompanhamento das publicações científicas, as principais conquistas, orientações e desafios da aplicação dessas estratégias em sala de aula; particularmente aqueles relacionados a finanças, administração e contabilidade.

Palavras-chave: Educação, Jogo sério, Administração, finanças e contabilidade, Aprendizado ativo, Treinamento por competencias.

\section{INTRODUCCIÓN}

Un desafío permanente en educación, que trasciende aspectos disciplinares y diferentes áreas del conocimiento, está asociado con la actualización de las didácticas y metodologías usadas en el aula de clase para lograr el aprendizaje activo y mejorar el logro de los objetivos académicos (Motiejunaite \& Žadeikaite, 2009).

De acuerdo con la generalidad de los casos actuales en educación superior, la enseñanza y el aprendizaje se logran cuando se verifica una respuesta adecuada del estudiante con base en el modelo de instrucción; así, la evaluación determina el cumplimiento de los estándares mínimos establecidos y permite identificar los reforzadores o estímulos que podrían ser más efectivos (Ertmer \& Newby, 1993). El desarrollo de actividades de enseñanza-aprendizaje y juegos serios en el aula de clase permite que los estudiantes desarrollen competencias laborales a partir de la simulación de escenarios, el estudio de casos y entornos multigrados (Abad et al., 2018).

Esto ha generado que se desarrollen acciones didácticas que promueven el mejoramiento de esa acción comunicativa alrededor de la educación, así como el desarrollo de la creatividad, la imaginación, la personalidad y la innovación en el plano cognitivo, psicológico y social (Von Graevenitz, Harhoff, \& Weber, 2010). 
Con base en estas acciones y su mezcla con herramientas asociadas a las Tecnologías de la Información y la Comunicación, TIC, se han desarrollado esquemas más complejos y sofisticados denominados comúnmente estrategias didácticas, que se expresan como un conjunto de métodos y secuencias definidas para alcanzar el logro de objetivos de enseñanza-aprendizaje, en los que se integran, en diferentes proporciones, discurso magistral, talleres, debates y foros con las consultas en línea, aulas virtuales, redes sociales, programas de diseño o simulación y videojuegos, y se logran nuevos modelos que comienzan a establecer tendencia en su aplicación (Lozano-Abad et al., 2019), resaltando que la incorporación de actividades didácticas dentro del aula de clase les permite a los estudiantes desarrollar diferentes habilidades y estrategias propias de cada estilo o tendencia de aprendizaje que le permiten afianzar el conocimiento (Ocampo et al., 2014).

\section{MARCO TEÓRICO}

El aprendizaje activo tiene dos acepciones, una en el campo de la educación y otra en el contexto informático. La primera está enfocada en una serie de estrategias didácticas que ubican al estudiante en el centro del proceso educativo, generando motivación que lo involucre de forma consciente en su función de aprendizaje y así establecer un vínculo con el conocimiento y su aplicación en contextos reales (Christie \& de Graaff, 2017). La segunda está centrada en el desarrollo de algoritmos de inteligencia artificial y aprendizaje de máquinas, con capacidad para realizar acciones creativas de búsqueda, comprobación, evaluación y almacenamiento de experiencias (Settles, 2010). Para efectos de este trabajo se usará la primera acepción.

Son diversas las iniciativas aplicadas en las aulas de clase y reportadas en la literatura científica; allí una de las vertientes reportada en las áreas de contabilidad, finanzas y gestión está asociada a la aplicación de los juegos en el proceso formativo (Bodnar \& Clark, 2017).

Entre otros fenómenos emergentes que procuran impactar positivamente el proceso de enseñanza - aprendizaje, y que van de la mano con la relevancia que han ganado las TIC en diferentes aspectos de la vida cotidiana, se pueden encontrar el concepto de micromundos, expuesto por Mavrikis et al., (2008) y Marín et al., (2015); la aplicación de juegos (Xinogalos et al., 2015) y la gamificación (Villalustre \& Del Moral, 2015), los cuales buscan convergencia hacia el aprendizaje significativo, incorporando en el proceso formativo un espacio de práctica simulada en el caso de micromundos y aplicación de juegos, y esquemas de estímulos y reconocimientos que eleven la motivación en el caso de gamificación (Acevedo, 2019).

El juego serio o formativo es una categoría que agrupa las aplicaciones del juego usadas en procesos educativos, los cuales no solo se aplican en los escuelas o universidades, sino que trascienden otras áreas como el entrenamiento militar, certificaciones en salud y simulaciones en el sector financiero para mejorar las destrezas de personas y equipos en 
entornos controlados. Se han desarrollado métodos para analizary diseñar juegos serios, y así evaluar la articulación de sus componentes con los objetivos educativos definidos (Carvalho et al., 2015). El juego formativo se ha abordado desde diferentes enfoques, entre ellos el cibernético, para comprenderlo como un proceso controlable y enfocado hacia los objetivos curriculares esperados (Westera, 2013).

Según Kark (2011), el juego y la gamificación han sido fundamentales en el desarrollo del aprendizaje, el cual es utilizado para obtener los resultados deseados durante el proceso de formación en estudiantes de diferentes niveles educativos; además, Pasin y Giroux (2011), mediante la implementación de un juego de simulación para la gestión de operaciones, muestran que este tipo de estrategias son más efectivas que las tradicionales y permiten el desarrollo de habilidades para la toma de decisiones de una manera más rápida. En campos como la medicina, utilizados en Europa (Garrido et al., 2018), se presentan hallazgos concluyentes por medio del uso de metodologías no convencionales, arrojando como resultados que los cursos que utilizan metodologías online y actividades de multimedia elevan su nivel de competencias de análisis y síntesis superiores a su nivel inicial (Flórez-Romero, R.\#; CastroMartinez, J. A.\#; Martelo Martínez, Fernando; Salazar-Montenegro, I.\#; Camelo-Cabuya, 2019)

De acuerdo con Sironi (2016), áreas como las finanzas y su creciente intersección con la tecnología (Fintech), deben direccionar también a las entidades educativas a evolucionar sus procesos académicos, adaptados a las TIC y estrategias creativas donde el estudiante se sienta motivado. Además, autores como Hamari, Huotari y Tolvanen (2015), destacan el uso de los juegos con fines de enseñar temas económicos y resaltan la atracción generada en docentes y estudiantes (Norman-Acevedo, 2019). Lo anterior puede ser respaldado por el experimento realizado a estudiantes de maestría en Economía de la Universidad de Granada, en la cual se implementó una plataforma de gamificación, donde se concluye que el uso de los juegos mejora la motivación en los estudiantes (Zamora \& Aranda, 2017).

\section{MÉTODO}

El desarrollo de este trabajo es de enfoque cualitativo, partiendo de los resultados de un estudio bibliométrico, mediante una ecuación de búsqueda en Scopus, donde se identificaron para la última década (2007-2018), características de cantidad, calidad y estructura de la producción académica y científica en la temática del aprendizaje basado en los juegos en programas de educación superior en las áreas de contabilidad, finanzas y administración. A partir de esta información se identifican las estrategias didácticas emergentes referidas por el Observatorio de Innovación Educativa del Tecnológico de Monterrey (Observatorio de Innovación Educativa, 2017), y se amplía la búsqueda a otras áreas del conocimiento, manteniendo como criterio amplio el aspecto educativo e ingenieril asociado al diseño y desarrollo de juegos serios, para identificar 
la frecuencia de aparición de esas estrategias didácticas emergentes en revistas y eventos arbitrados y aprobados por la comunidad científica.

Con base en lo anterior se indaga en los textos de mayor relevancia e índice de impacto para describir aspectos que destaquen, como el perfil de los participantes, el alcance, la relación con el juego serio y, finalmente, los principales retos o desafíos en cada una de estas estrategias didácticas emergentes que se logran identificar en la revisión de literatura.

\section{RESULTADOS}

Bibliometria: los resultados obtenidos del estudio bibliométrico muestran que el incremento de la producción académica sobre esta temática es significativo y cada año se incrementa a razón del $10 \%$ según la tendencia; durante la década 2007-2018 se lograron hallar 816 registros. Esto indica que el interés en la temática es creciente y consistente con procesos de transformación digital que se están viviendo en otros ámbitos como la tecnificación, la producción y las finanzas.

Los trabajos en formato artículo ocupan un cuarto de la producción total, mientras que los textos de conferencias son predominantes, superando el $50 \%$ de la producción académica indexada, señalando un amplio interés en la difusión, principalmente de documentos reflexivos y de carácter aplicado a contextos específicos. En su gran mayoría son trabajos realizados por dos o tres autores.

De otra parte, el foco de producción en esta temática está en los países desarrollados: allí se encuentran Estados Unidos, Reino Unido, China, Alemania, España, Canadá, Holanda, entre otros. Dentro de las universidades que han aportado ocho trabajos o más en esta temática durante la última década están Delft University of Technology, de Holanda; University of Ontario Institute of Technology, de Canadá; Universidad Complutense de Madrid, en España; National Central University Taiwan, de China, y CNRS Centre National de la Recherche Scientifique, de Francia.

Entre los autores y trabajos más representativos durante esta década, con más de 100 citaciones, están Proserpio y Gioia, con un trabajo de revisión orientado a enseñar a la generación de la virtualidad, como iniciativa colaborativa entre las universidades Bocconi, en Italia, y Estatal de Pennsylvania, en Estados Unidos, el cual aporta elementos de referencia para el diseño de objetos y ambientes virtuales; es citado por diferentes autores como un manual para el diseño de actividades de aprendizaje (Proserpio \& Gioia, 2007).

Tendencias: como parte de este análisis, y tomando como referencia la tipología propuesta por el Observatorio de Innovación Educativa del Tecnológico de Monterrey (Observatorio de Innovación Educativa, 2017), se detectan en la literatura asociada al aprendizaje basado en juegos algunas estrategias didácticas emergentes que describen tendencia en el discurso de la educación y el aprendizaje, dando enfoque a comunidades académicas que, desde diferentes países y universidades, se agrupan en torno a cada temática. Entre ellas encontramos: "aprendizaje 
colaborativo" (collaborative-based learning), “aprendizaje basado en retos" (challenge-based learning), "aprendizaje adaptativo" (adaptative learning), "aprendizaje invertido" (fipped learning), educación basada en competencias (Competency(skill)-based education), Massive Open Online Course (MOOC), gamificación (gamification) y narrativas (storytelling).

Luego de tener clasificadas las iniciativas en estas ocho categorías, se procede a identificar el impacto de acuerdo con una proporción razonable de trabajos, número de citaciones, uso y alcance descrito por los autores más relevantes en torno a la aplicación y resultados. A partir de esta proporción se logra determinar tres categorías:

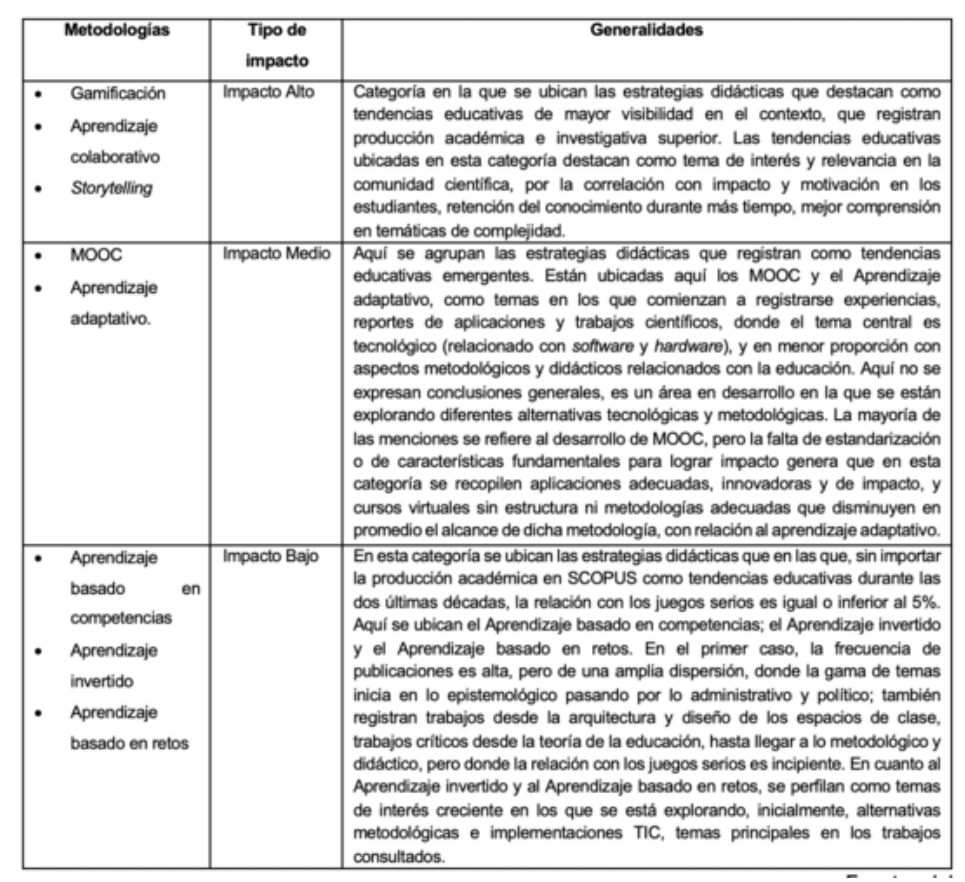

Tabla 1.

Frecuencia de registro Tendencias

Fuente: elaboración propia, a partir de información obtenida de Scopus, 2019.

Retos: esta descripción surge de una construcción conceptual propia de los resultados obtenidos en el estudio bibliométrico; las tendencias contienen información relacionada con: la definición, la asociación de cada tendencia con el uso de los juegos, la relación de cada tendencia con las ciencias económicas, el papel de estudiantes y docentes, además, de los desafíos y retos presentes en su implementación. 


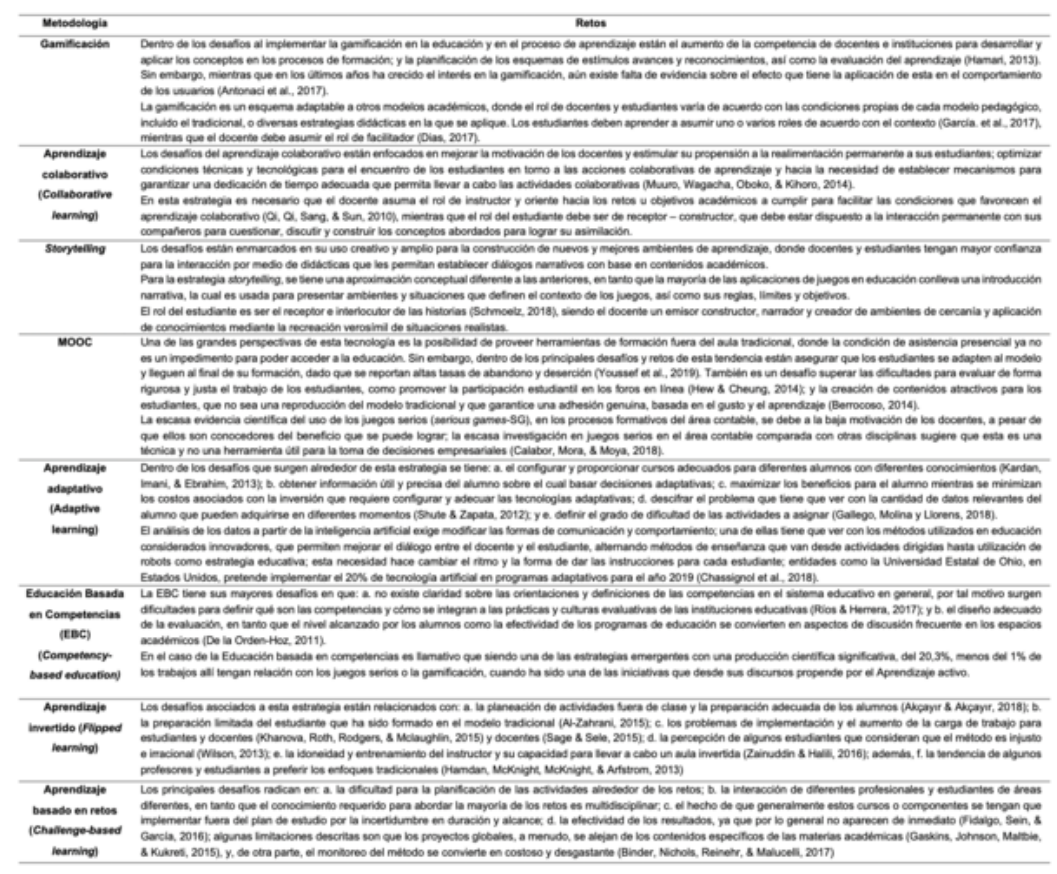

Tabla 2.

Metodología y Retos.

Fuente: elaboración propia, 2019.

\section{CONCLUSIONES}

La actualización curricular y didáctica es un tema de interés exploratorio para la academia en diferentes áreas disciplinares, el cual ha comenzado a salir de los recintos de las facultades de educación para convertirse en responsabilidad de las profesiones que deben resolver la optimización de los procesos formativos. Es importante mencionar que la interdisciplinariedad no es posible sí las áreas no se atreven a incursionar en los campos de otras, con el genuino interés de mejorar los procesos formativos que son de competencia propia o donde se interceptan los campos disciplinares.

La educación actual invita a revisar procesos metodológicos motivando en los docentes la realización de cambios significativos en sus procesos de enseñanza, entregando al estudiante un rol protagónico, al hacer parte fundamental de su proceso formativo; la inclusión de herramientas diferentes al aprendizaje convencional facilita el proceso de enseñanza, permitiendo a las generaciones de la era tecnológica vivir experiencias significativas en el fortalecimiento de competencias, especialmente las blandas; por el desarrollo de algunas de ellas como el autocontrol, trabajo colaborativo y comunicación que les exige la realización de trabajos independientes guiados por su tutor, en entornos diferentes a las aulas de clase (Trujillo-Flórez et al., 2019).

La gamificación muestra ser la estrategia con mayor alcance e impacto, debido a su capacidad de adaptación con otras estrategias y a la generación de procesos mentales basados en imágenes gráficas que fijan los conceptos en la memoria y que se relacionan con recuerdos agradables y divertidos 
asociados a la interacción producida por el juego como expresión social. Sin embargo, aún es relevante la apreciación de algunos autores y experimentos como herramienta educativa, lo que resta credibilidad al proceso y que genera dispersión en los actores (Daza-Orozco, 2015; Trujillo-Flórez et al., 2018; Velandia Vargas et al., 2019).

Las herramientas pedagógicas de enseñanza-aprendizaje resultan aportantes al proceso académico en áreas financieras, económicas y administrativas, una vez que se dé el desarrollo de contenidos que representan situaciones replicables, tomas de decisiones y aplicaciones matemáticas y estadísticas.

A pesar de que esta revisión está direccionada al campo administrativo y financiero, la aplicación de estas estrategias no es excluyente. Se presenta un acercamiento a la generalización para aportar a la innovación educativa desde diferentes visiones.

\section{DISCUSIÓN}

Aunque en este documento se presentan algunas estructuras, estas no son las únicas que se han explorado en las áreas educativas propuestas y/ o en otras áreas. Se han investigado diferentes esquemas para fomentar la eficiencia y la eficacia de la educación en el mundo y el logro de competencias básicas de los estudiantes. Por ejemplo, desde organismos de cooperación internacional como la OCDE, se lidera el Programa Internacional de Evaluación de Estudiantes, PISA (Vaillant \& Zidán, 2016), donde se busca medir el rendimiento de los estudiantes en diferentes áreas, lo que ha suscitado en algunos países el modelo de medición objetivo en diferentes niveles del proceso educativo. Otros fenómenos más locales avanzan en tendencias metodológicas que pueden lograr resultados exitosos, como lo es el Aprendizaje Basado en Proyectos, según lo indican Prince y Felder (2006), y variaciones como el Aprendizaje Basado en Problemas y Retos tal como lo menciona Hmelo (2004), siguiendo las lecciones aprendidas y buenas prácticas en países como Finlandia, Singapur y Corea del Sur. En otros casos se han implementado innovaciones pedagógicas más sutiles, pero de menor radicalidad, como las transformaciones curriculares en la reorganización de contenidos que no afectan el modelo metodológico ni el evaluativo. De igual manera, Pico et al. (2018) y Villa et al. (2020), presentan una importante reflexión desde las ventajas y las desventajas de usar estrategias de enseñanza-aprendizaje, aclarando la importancia de enmarcar los retos presentados en contextos a partir de concepciones culturales y capacitaciones docentes como base para mejorar los alcances. De igual manera, se plantea identificar la diferenciación de campus presencial y campus digital, y el papel del formador en el uso de las herramientas informáticas.

Para ampliación de este trabajo o trabajos futuros, se recomienda la revisión de otros conceptos asociados y validados en casos de estudio específico, que puedan aportar más herramientas para la construcción de una educación de calidad y adaptativa a contextos diversos. 


\section{REFERENCIAS}

Akçayır, G., \& Akçayır, M. (2018). The flipped classroom: A review of its advantages and challenges. Computers and Education, 126, 334-345. htt ps://doi.org/10.1016/j.compedu.2018.07.021

Al-Zahrani, A. (2015). From passive to active: The impact of the flipped classroom through social learning platforms on higher education students' creative thinking. British Journal of Educational Technology, 46(6), 1133-1148. https://doi.org/10.1111/bjet.12353

Berrocoso, J. (2014). MOOCS: A critical view from the Education Sciences. Profesorado, 18(1), 93-111. https://doi.org/10.3916/C41-2013-a1; Alba Pastor, C., Aportaciones del Diseño Universal para el Aprendizaje y de los materiales digitales en el logro de una enseñanza accesible (2012) Respuestas Flexibles en Contextos Educativos Diversos, pp. 1-13. , http://diversidad.murciaeduca.es/publicaciones/dea2012/docs/ calba.pdf, J. Navarro, M. T. Fernández, F. J. Soto, \& F. Tortosa (Eds.) Murcia: Región de Murcia. Consejería de Educación, Formación y Empleo; Anderson, T., Rourke, L., Garrison, D.R., Archer, W., Assessin

Binder, F., Nichols, M., Reinehr, S., \& Malucelli, A. (2017). Challenge Based Learning Applied to Mobile Software Development Teaching. Proceedings - 30th IEEE Conference on Software Engineering Education and Training, CSEE and T 2017. https://doi.org/10.1109/CSEET.2017 .19

Bodnar, C. A., \& Clark, R. M. (2017). Can game-based learning enhance engineering communication skills? IEEE Transactions on Professional Communication, 60(1), 24-41. https://doi.org/10.1109/TPC.2016.263 2838

Calabor, M., Mora, A., \& Moya, S. (2018). The future of "serious games" in accounting education: A Delphi study. Journal of Accounting Education. https://doi.org/10.1016/j.jaccedu.2018.12.004

Carvalho, M. B., Bellotti, F., Berta, R., De Gloria, A., Sedano, C. I., Hauge, J. B., ... Rauterberg, M. (2015). An activity theory-based model for serious games analysis and conceptual design. Computers and Education. https:/ /doi.org/10.1016/j.compedu.2015.03.023

Chassignol, M., Khoroshavin, A., Klimova, A., \& Bilyatdinova, A. (2018). Artificial Intelligence trends in education: a narrative overview. Procedia Computer Science. https://doi.org/10.1016/j.procs.2018.08.233

Christie, M., \& de Graaff, E. (2017). The philosophical and pedagogical underpinnings of Active Learning in Engineering Education. European Journal of Engineering Education. https://doi.org/10.1080/03043797.2 016.1254160

Daza-Orozco, CE. (2015). Investigación y emprendimiento: Experiencias de las Instituciones de Educación Superior Mesa IEST. Corporación Internacional para el desarrollo Educativo - CIDE. https://doi.org/10.13 140/RG.2.1.4728.3283

De La Orden Hoz, A. (2011). The competency problems in general education | El problema de las competencias en la educación general. Bordon.

Ertmer, P., \& Newby, T. (1993). Behaviorism, Cognitivism, Constructivism: Comparing Critical Features from an Instructional Design Perspective. 
Performance Improvement Quarterly, 6(4), 50-72. https://doi.org/10.1 $111 /$ j.1937-8327.1993.tb00605.x

Fidalgo, A., Sein, M., \& García, F. (2016). Integration of the methods CBL and CBI for their application in the management of cooperative academic resources. 2016 International Symposium on Computers in Education, SIIE 2016: Learning Analytics Technologies. https://doi.org/10.1109/S IIE.2016.7751849

Flórez-Romero, R.\#; Castro-Martinez, J. A.\#; Martelo Martínez, Fernando; Salazar-Montenegro, I.\#; Camelo-Cabuya, R. R.\#; C. M. (2019). El juego: un asunto serio en la formación de los niños y las niñas (1st ed.). Bogotá: Institución Universitaria Politécnico Grancolombiano.

Gallego, F., Molina, R., \& Llorens, F. (2018). Measuring the difficulty of activities for adaptive learning. Universal Access in the Information Society. https://doi.org/10.1007/s10209-017-0552-x

Garrido, M., Santiago, G., Márquez, M., Poggio, L., \& Gómez, S. (2018). The impact of digital resources in the learning and the development of the competence Analysis and Synthesis. Educacion Medica, (xx). https://doi .org/10.1016/j.edumed.2018.02.011

Gaskins, W., Johnson, J., Maltbie, C., \& Kukreti, A. (2015). Changing the Learning Environment in the College of Engineering and Applied Science Using Challenge Based Learning. International Journal of Engineering Pedagogy. https://doi.org/10.3991/ijep.v5i1.4138

Hamari, J., Huotari, K., \& Tolvanen, J. (2015). Gamification and Economics. In The Gameful World (pp. 139-161). https://doi.org/10.7551/mitpres s/9788.003.0009

Hamdan, N., McKnight, P., McKnight, K., \& Arfstrom, K. (2013). A Review of Flipped Learning. Flipped Learning Network, 20. https://doi.org/10.1 016/j.compedu.2015.07.008

Hew, K., \& Cheung, W. (2014). Students' and instructors' use of massive open online courses (MOOCs): Motivations and challenges. Educational Research Review, Vol. 12, pp. 45-58. https://doi.org/10.1016/j.edurev.2 014.05 .001

Hmelo, C. (2004). Problem-based learning: What and how do students learn? Educational Psychology Review, 16(3), 235-266. https://doi.org/10.102 3/B

Kardan, A., Imani, M., \& Ebrahim, M. (2013). A novel adaptive learning path method. 4th International Conference on E-Learning and e-Teaching, ICELET 2013. https://doi.org/10.1109/ICELET.2013.6681639

Kark, R. (2011). Games managers play: Play as a form of leadership development. Academy of Management Learning and Education, 10(3), 507-527. https://doi.org/10.5465/amle.2010.0048

Khanova, J., Roth, M., Rodgers, J., \& Mclaughlin, J. (2015). Student experiences across multiple flipped courses in a single curriculum. Medical Education, 49(10), 1038-1048. https://doi.org/10.1111/medu.12807

Lozano-Abad, Y. C., Rosales-Doria, A. M., \& Giraldo-Cardozo, J. C. (2019). COMPETENCIAS DEL SIGLO XXI: ¿CÓMO DESARROLLARLAS MEDIANTE EL USO DE VIDEOJUEGOS EN UN CONTEXTO MULTIGRADO? PANORAMA, 12(23), 6. https://doi.org/10.15765/ pnrm.v12i23.1191 
Marín, Y., Posada, W., García, B., \& Munévar, R. (2015). Metodología para la creación de micromundos interactivos. Methodology for the Creation of Interactive MicroWorlds., 12(11), 61-81.

Mavrikis, M., Geraniou, E., Noss, R., \& Hoyles, C. (2008). Revisiting pedagogic strategies for supporting students' learning in mathematical microworlds. devolving teachers' role to an "intelligent" facilitator. In CEUR Workshop Proceedings (Vol. 381).

Motiejunaite, E., \& Žadeikaite, L. (2009). Competences development\#: Challenges and possibilities. Pedagogika, 95, 86-93.

Muuro, M. E., Wagacha, W. P., Oboko, R., \& Kihoro, J. (2014). Students' Perceived Challenges in an Online Collaborative Learning Environment: A Case of Higher Learning Institutions in Nairobi, Kenya. International Review of Research in Open and Distance Learning.

Norman-Acevedo, E. (2019). Nuevos lenguajes para aprendizaje virtual herramientas para los escenarios de aprendizaje. Revista Panorama, 24(13), 5-7. https://doi.org/http://dx.doi.org/10.15765/pnrm.v13i24. 1214

Observatorio de Innovación Educativa. (2017). Radar de Innovación educativa 2017. In Reporte Edu Trends. ITESM. México.

Ocampo Botello, F., Guzmán Arredondo, A., Camarena Gallardo, P., \& De Luna Caballero, R. (2014). Identificación de estilos de aprendizaje en estudiantes de ingeniería. Revista Mexicana de Investigacion Educativa.

Pasin, F., \& Giroux, H. (2011). The impact of a simulation game on operations management education. Computers and Education, 57(1), 1240-1254. h ttps://doi.org/10.1016/j.compedu.2010.12.006

Prince, M. J., \& Felder, R. M. (2006). Inductive teaching and learning methods: Definitions, comparisons, and research bases. Journal of Engineering Education, 95(2), 123-138. https://doi.org/10.1002/j.2168-9830.2006. tb00884.x

Proserpio, L., \& Gioia, D. A. (2007). Teaching the virtual generation. Academy of Management Learning and Education, 6(1), 69-80. https://doi.org/1 0.5465/AMLE.2007.24401703

Qi, C., Qi, Z., Sang, J., \& Sun, Y. (2010). Web-based collaborative learning system. 2010 International Conference on Networking and Digital Society. https://doi.org/10.1109/ICNDS.2010.5479441

Ríos, D., \& Herrera, D. (2017). Los desafíos de la evaluación por competencias en el ámbito educativo. Educ. Pesqui. https://doi.org/10.1590/S1678-46 34201706164230

Sage, M., \& Sele, P. (2015). Reflective Journaling as a Flipped Classroom Technique to Increase Reading and Participation with Social Work Students. Journal of Social Work Education, 51(4), 668-681. https://do i.org/10.1080/10437797.2015.1076274

Schmoelz, A. (2018). Enabling co-creativity through digital storyelling in education. Thinking Skills and Creativity. https://doi.org/10.1016/j.tsc. 2018.02.002

Settles, B. (2010). Active Learning Literature Survey. Machine Learning. https ://doi.org/10.1.1.167.4245 
Shute, V., \& Zapata, D. (2012). Adaptive educational systems. Adaptive Technologies for Training .... https://doi.org/10.1017/CBO978113904 9580.004

Sironi; Paolo. (2016). FinTech innovation: from robo-advisors to goal based investing and gamification. In John Wiley \& Sons. https://doi.org/10.10 02/9781119227205.ch1

Trujillo-Flórez, L. M., Bernal Yermanos, M. Á., Escobar Castro, J. A., González Triana, M. C., Gutiérrez Carvajal, J. P., Gutiérrez Vanegas, J. C., ... Torres López, A. L. (2019). Experiencias de innovación educativa Tomo 3 (1st ed.; Luis Martín Trujillo-Flórez, Ed.). Bogotá: Politécnico Grancolombiano.

Trujillo-Flórez, Luis Martín;, Martínez Contreras, R. M., Espitia López, H., Rojas Paredes, J. C., Vargas Leguizamón, Y. R., \& Castro Cabal, G. A. (2018). Experiencias de innovación educativa (1st ed.; L. M. FlórezTrujillo, Ed.). Bogotá: Politécnico Grancolombiano.

Vaillant, D., \& Zidán, E. R. (2016). Leadership practices for learning in Latin America: Analysis from PISA 2012. Ensaio: Avaliação e Políticas Públicas Em Educação, 24(91), 253-274. https://doi.org/10.1590/S0104-40362 016000200001

Velandia Vargas, G. K., Malangón Torres, M. A., Ramos Duarte, N., Quiroz Rubiano, M. M., Rodríguez Rubio, A., \& Celemín Pabón, Y. A. (2019). Experiencias de innovación educativa - Tomo 2 (1st ed.; Luis Martín Trujillo-Flórez, Ed.). Bogotá: Politécnico Grancolombiano.

Villalustre Martínez, L., \& Del Moral Peréz, M. (2015). Gamitication: Strategies to optimize learning process and the acquisition of skills in university contexts. Digital Education Review, (27), 13-31.

von Graevenitz, G., Harhoff, D., \& Weber, R. (2010). The effects of entrepreneurship education. Journal of Economic Behavior and Organization, 76(1), 90-112. https://doi.org/10.1016/j.jebo.2010.02.01 5

Westera, W. (2013). On the cybernetic arrangement of feedback in serious games: A systems-theoretical perspective. Education and Information Technologies. https://doi.org/10.1007/s10639-013-9267-7

Wilson, S. (2013). The Flipped Class: A Method to Address the Challenges of an Undergraduate Statistics Course. Teaching of Psychology, Vol. 40, pp. 193-199. https://doi.org/10.1177/0098628313487461

Xinogalos, S., Malliarakis, C., Tsompanoudi, D., \& Satratzemi, M. (2015). Microworlds, Games and Collaboration Three effective approaches to support novices in learning programming. https://doi.org/10.1145/2801 081.2801094

Youssef, M., Mohammed, S., Hamada, E. K., \& Wafaa, B. F. (2019). A predictive approach based on efficient feature selection and learning algorithms' competition: Case of learners' dropout in MOOCs. Education and Information Technologies, 1-28. https://doi.org/10.1007/s10639-01909934-y

Zainuddin, Z., \& Halili, S. H. (2016). Flipped classroom research and trends from different fields of study. International Review of Research in Open and Distance Learning, 17(3), 313-340. https://doi.org/10.19173/irrod l.v17i3.2274 
Zamora, J. C. F., \& Aranda, D. A. (2017). Implementation of a Gamification Platform in a Master Degree (Master in Economics). Working Papers on Operations Management, 8, 181-190. https://doi.org/http://dx.doi.org /10.4995/wpom.v8i0.7431

\section{Información adicional}

Para citar este articulo: / To cite this article: / Para citar este artigo:: Morales, M., Cardona-Valencia, D., Castañeda-Gómez, E., Uribe-Ortiz, A., Ríos-Gallego, P. (2020). APLICACIÓN DEL JUEGO SERIO EN PROGRAMAS DE CIENCIAS ECONÓMICAS: TENDENCIAS Y DESAFÍOS (Politécnico Grancolombiano, Ed.). Revista Panorama, 14(27), DOI: http://dx.doi.org/10.15765/pnrm.v14i27.1526 